\title{
Abracadabra! Replication shutdown
}

DOI:

10.1038/nrmicro1666

URLs

Bacillus subtilis

http://www.ncbi.nlm.nih.gov/ entrez/query.fcgi?db=genome prj\&cmd=Retrieve\&dopt=Over viewElist_uids=17579

Escherichia coli http://www.ncbi.nlm.nih.gov/ entrez/query.fcgi?db=genome prj\&cmd=Retrieve\&dopt=Over viewElist_uids=12319
When bacteria are starved, the stringent response coordinates cell functions to divert scarce resources from growth and cell division to amino-acid biosynthesis in order to aid survival. Publishing in Cell, Wang et al. now show that the stringent response shuts down genome replication in Bacillus subtilis and conclude that signals deployed during the stringent response directly inhibit DNA primase, an essential part of the DNA replication machinery.

The stringent response is mediated by the small guanosine nucleotides (p)ppGpp - ppGpp and pppGpp - that are collectively known as alarmones or magic spots. Upon binding to the RNA polymerase, (p)ppGpp inhibits the transcription of genes that encode the translation machinery and promotes the transcription of genes that encode amino-acid biosynthetic pathways. In Escherichia coli the stringent response also inhibits the initiation of genome replication, thereby shifting the focus of cellular activity from cell division to cell survival.

In 1991, work from Simone Séror's laboratory first demonstrated that, in B. subtilis, the stringent response regulates replication elongation rather than initiation. Unlike the mechanisms underlying the initiation of DNA replication in bacteria, those controlling elongation of replication are not well understood. Replication of the lone circular B. subtilis chromosome initiates from a single origin of replication (ori) and proceeds bidirectionally around the chromosome. Subsequent work revealed that starvation results in an abrupt shutdown of chromosome replication at specific left and right stringent termination (ter) sites located either side of the ori.

In the latest report, Wang et al. used time-resolved whole genome microarrays to monitor the movement of replication forks in synchronized cell cultures by pinpointing the doubling of DNA content at individual gene loci. When B. subtilis cells were starved, replication forks stalled at the ter sites in accordance with previous findings. But replication forks also stalled within minutes - independent of their location - when an asynchronous population was starved. This argues against the presence of dedicated cis-acting sites at which elongation can be terminated.

RecA is usually recruited to repair chromosomes when replication forks stall in bacteria, but the authors found that this did not happen in the stringent response of $B$. subitilis. In fact, the authors showed that stalled forks can restart replication when nutrients become available, and argue that rapidly stalling replication prevents the accumulation of doublestrand breaks that might arise from genome replication in the absence of sufficient dNTPs.

The authors sought to probe how starvation and chromosome replication are coordinated. The obvious candidate was the replication termination (Rtp) protein, but replication forks stalled in response to starvation in a mutant that lacked Rtp. A mutant lacking RelA, which is a (p)ppGpp synthetase that is activated in the stringent response, did not terminate replication when cells were starved. A suite of experiments revealed that (p)ppGpp targets DNA primase, an RNA polymerase that is dedicated to priming DNA replication. Just where (p)ppGpp binds to DNA primase is not yet known but the mechanism of inhibition is sensitive, because gradually increasing (p)ppGpp concentrations switches off elongation in a dose-dependent fashion. The response can therefore be tuned according to the extent of nutrient limitation.

This study provides an elegant link between nutrient availability and the preservation of genome integrity and might form part of a general bacterial stress response to ensure survival in adverse conditions.

\section{Susan Jones}

ORIGINAL RESEARCH PAPER Wang, J. D. Sanders, G. M. \& Grossman, A. D. Nutritional control of elongation of DNA replication by (p)ppGpp. Cell 128, 865-875 (2007)

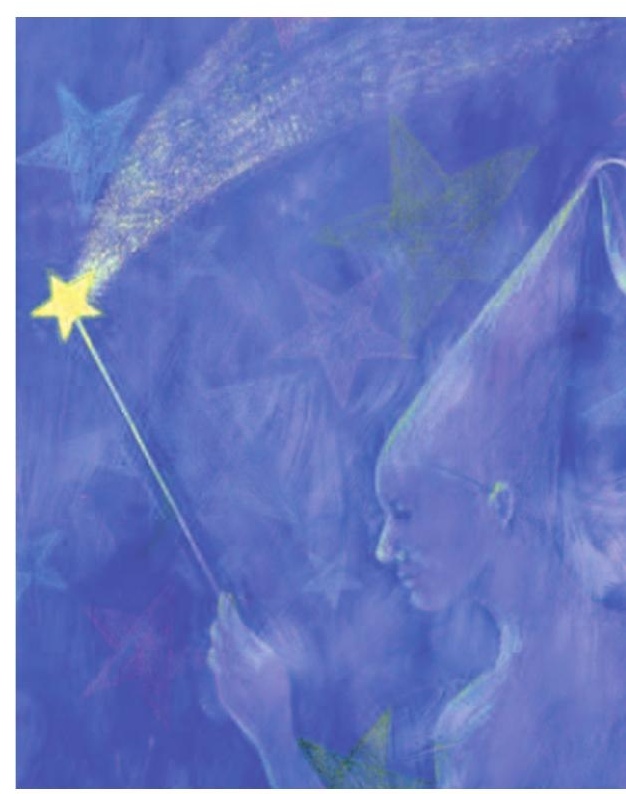

